\title{
AVALIAÇÃO DO TEOR DE ÓLEO ESSENCIAL DE Rosmarinus sp. CULTIVADO EM DOIS NÍVEIS DE LUMINOSIDADE.
}

OIL CONTENT OF ASSESSMENT OF ESSENTIAL Rosmarinus sp. GROWN IN TWO LEVELS SHADOWING.

\section{Alyne Rodrigues de Moura}

Acadêmica do curso de Farmácia, Facer- Faculdade Evangélica de Ceres, Ceres-GO, Brasil. alynerodrigues89@hotmail.com;

\section{Isamara Cintra Medeiros}

Acadêmica do curso de Farmácia, Facer- Faculdade Evangélica de Ceres, Ceres-GO, Brasil. isamara.cm@hotmail.com;

\section{Maria Juíva Marques de Faria Souza}

Professora do curso de Farmácia, Facer- Faculdade Evangélica de Ceres, Ceres-GO, Brasil.juivamaria@hotmail.com.

\section{RESUMO}

Introdução: Rosmarinus sp. (Lamiaceae) conhecido como alecrim é um arbusto aromático. Estudos científicos vêm demonstrando suas ações estimulantes, antiespasmódicas, cicatrizantes, antiinflamatórias, antitumorais e quimiopreventivas. Ressalta-se que suas propriedades estão relacionadas com metabólitos secundários como os terpenos, flavonóides e ácidos fenólicos. Há vários fatores que podem interferir no rendimento dos metabólitos secundários, a luz é um destes. Objetivo: Avaliar o teor de óleo essencial da espécie Rosmarinus sp. cultivadas em dois níveis de luminosidade (30\% de luminosidade e diretamente ao sol). Metodologia: Antes de iniciar o tratamento, o óleo essencial de três mudas de alecrim foi extraído em triplicata pelo método de hidrodestilação acoplado ao aparato de Clevenger, por 2 horas, com banho de refrigeração à $25^{\circ} \mathrm{C}$. Logo, os demais alecrins foram divididos em dois grupos experimentais (sombreamento com $30 \%$ de luminosidade e o outro a pleno sol) por 30 dias. Posterior a isso, as mudas foram submetidas a novas extrações utilizando o mesmo método. $\mathrm{O}$ teor foi expresso em porcentagem de óleo essencial (\%) em relação à massa fresca. Resultados e Discussão: Antes do tratamento o teor do óleo essencial foi 3,33\%, e após os tratamentos a pleno sol e com $30 \%$ de luminosidade o resultado foi o mesmo. Conclusão: Não houve diferença significativa no teor de óleo essencial das plantas cultivadas a pleno sol e com as plantas cultivadas a sombreamento de $30 \%$ de luminosidade. Devido à importância dos óleos essenciais e seus valores econômicos no Brasil é importante continuar o estudo sugerindo aumentar o tempo do tratamento.

Palavras chave: Alecrim. Hidrodestilação. Luminosidade. 


\begin{abstract}
Introduction: Rosmarinus sp. (Lamiaceae) known as Rosemary is an aromatic shrub. Scientific studies have demonstrated its stimulating action, antispasmodic, healing, anti-inflammatory, antitumor and chemopreventive. It is noteworthy that its properties are related secondary metabolites such as terpenes, flavonoids and phenolic acids. There are several factors that can interfere with the performance of secondary metabolites, light is one of these. Objective: To evaluate the essential oil content of the species Rosmarinus sp. grown at two levels of luminosity (30\% light and direct sunlight). Methodology: Before you start treatment rosemary essential oil was extracted in triplicate by hydrodistillation method coupled to apparatus Clevenger, for 2 hours with cooling bath at $25^{\circ} \mathrm{C}$. The content was expressed in essential oil percentage (\%) compared to fresh pasta. Soon, rosemary was divided into two experimental groups (shading with $30 \%$ brightness and the other full sun) for 30 days. Subsequent to this, the seedlings were subjected to further extractions using the same method. Results and Discussion: Before treatment the essential oil content was $3.33 \%$, after treatments in full sun and $30 \%$ light there were no significant changes. Conclusion: There was no significant difference in the essential oil content of plants grown in full sun and plants grown shading $30 \%$ of brightness due to the importance of essential oils and their economic values in Brazil continuity of the study with longer experiment is feasible.
\end{abstract}

Keywords: Rosemary. Hydrodistillation. Brightness.

\title{
Endereço para correspondência:
}

Av. Brasil, S/N, Qd. 13, Setor Morada Verde Ceres - Go. CEP: 763000-000

Fone: (62) 3323-1040

juivamaria@hotmail.com

\section{INTRODUÇÃO}

O alecrim (Lamiaceae) é um arbusto aromático muito utilizado na terapêutica e como condimento (CLEFF et al., 2012; FRESCURA et al., 2013). Estudos científicos vêm demonstrando suas propriedades benéficas como estimulante digestivo, antiespasmódica, emenagoga, cicatrizante, antiinflamatória, antitumoral e quimiopreventiva (MAY et al., 2010; AFONSO et al., 2010). Possui também ação antimicrobiana, principalmente frente aos gêneros Staphylococcus, Streptococcus, Proteus, Pseudomonas, Klebsiella, Salmonella, Mycobacterium e Escherichia (CLEFF et al., 2012).

Ressalta-se que essas propriedades estão relacionadas com a presença dos metabólitos secundários, os quais têm a função biológica de garantir vantagens para a sobrevivência e continuidade das espécies vegetais, e do ponto de vista farmacológico fonte para o 
desenvolvimento de novos fármacos (SIMÕES et al., 2007). Os principais metabólitos secundários encontrados no alecrim são: hidrocarbonetos monoterpênicos, ésteres terpênicos, linalol, verbitol, terpineol, 3-octonona, acetato de isobornila, cânfora, 1,8-cineol, $\alpha$-pineno, verbenona, borneol, os quais são constituintes do óleo essencial (RIBEIRO et al., 2012). Também são encontrados quercetina, rutina e canferol (flavonóides) e ácido clorogênico, ácido caféico, ácido rosmarínico e ácido carnósico (ácidos fenólicos) (FRESCURA et al., 2013).

O óleo essencial do alecrim é muito usado na indústria, principalmente na perfumaria, cosmética e na área de alimentos (MORAIS, 2009). Logo, o valor econômico do alecrim é conferido ao aspecto dos óleos essenciais, que provêm das folhas e flores, atribuindo forte odor canforáceo e sabor claramente fresco e picante (PRINS et al., 2008). Estima-se que para obtenção dos óleos essenciais de espécies da família Lamiaceae como o alecrim, mais de 500 mil hectares são usados para o cultivo dessas plantas (SIMÕES et al., 2007).

A formação dos óleos essenciais das plantas pode ser definida e alterada conforme a espécie, órgão de um vegetal, multiplicidade genética, tempo de coleta, modo de processar (TEIXEIRA, 2012), solo, umidade e exposição ao sol (MULLER, 2013).

A luz, fonte primária de energia, esta ligada diretamente com a fotossíntese, que é um dos principais fatores que influenciam no desenvolvimento dos vegetais. A resposta ao fator luz é característica específica de cada espécie (CAMPOS; UCHIDA, 2002). Portanto, a luz é parte fundamental para desenvolvimento do vegetal, caracterizada como um dos principais fatores que controla o crescimento, desenvolvimento e o metabolismo (MEIRA et al., 2012).

Isso pode ser observado no estudo realizado por Chagas (2009), em que plantas da espécie Mentha arvensis L. (hortelã-japonesa), da mesma família que o alecrim, quando cultivada a pleno sol observou-se um maior rendimento de óleo essencial do que as plantas cultivadas em sombreamento a 50\%. Em contrapartida, no estudo desenvolvido por Araújo (2014) o maior rendimento de óleo essencial Tetradenia riparia (falsa mirra) ocorreu nas plantas cultivadas nos tratamentos de $30 \%$ e $50 \%$ de sombreamento do que a pleno sol.

Diante do exposto o objetivo deste estudo foi avaliar o teor de óleo essencial da espécie Rosmarinus sp. cultivadas em dois níveis de luminosidade (30\% de luminosidade e a pleno sol).

\section{METODOLOGIA}

Foi realizada uma pesquisa experimental quantitativa, sendo o objeto de estudo a espécie Rosmarinus sp. (alecrim). As 13 mudas de alecrim foram obtidas em julho de 2015 no viveiro 
Castro Floricultura na cidade de Itapuranga-GO, e em seguida cultivadas na Faculdade de Ceres (Facer), localizada na cidade de Ceres-GO.

Durante 30 dias as mudas foram cultivadas em sacos plásticos para cultivos de plantas no tamanho de $12 \times 12 \mathrm{~cm}$, logo para melhor desenvolvimento foram transplantadas para sacos plásticos de $11 \times 20$.

Com o objetivo de verificar o possível aumento do teor do óleo essencial Rosmarinus sp., e este sendo verídico, antes de iniciar os tratamentos foram realizadas extrações por hidrodestilação em aparato do tipo Clevenger por 2 horas (BRASIL, 2010; BARBOSA, et al., 2014), em triplicata de três mudas diferentes com o intuito de analisar se o aumento estaria relacionado com qual nível de luminosidade em que as plantas foram cultivadas.

Para o processo de extração, as folhas de Rosmarinus sp. foram coletadas e introduzidas no balão de destilação juntamente com água destilada, o qual foi mantido em uma manta de aquecimento com temperatura máxima de $100^{\circ} \mathrm{C}$ até atingir fervura. Em seguida, o balão foi acoplado ao aparelho de Clevenger, com o banho de refrigeração à $25^{\circ} \mathrm{C}$. Devido à fotossensibilidade do óleo essencial todo o aparelho foi envolvido com papel alumínio.

Em seguida separou os dois grupos de tratamento, 05 mudas foram cultivadas a pleno sol e 05 mudas foram cultivadas debaixo de um sombrite a $30 \%$ de luminosidade por 30 dias. Essas mudas não foram adicionadas adubo ou vitaminas.

Após o tratamento o óleo essencial da espécie em estudo foi extraído novamente, conforme o método descrito anteriormente. Logo, realizou extração em triplicata para cada grupo experimental.

Os resultados referentes ao rendimento de óleo essencial das extrações de cada tipo de sombreamento foram expressos em teor de óleo essencial (\%) em relação à massa fresca. Os dados adquiridos foram submetidos a análise de variância, e as médias foram comparadas pelo Teste $\mathrm{T}$ a $5 \%$ de probabilidade.

\section{RESULTADOS E DISCUSSÃO}

Antes de iniciar os tratamentos realizou-se a extração por hidrodestilação acoplado ao aparelho Clevenger em triplicata da espécie em estudo para verificar se a possível variação do rendimento do teor de óleo essencial estaria relacionada com as mudas cultivadas em pleno sol ou a $30 \%$ de luminosidade. Portanto, a média do rendimento foi 3,33\% de óleo essencial, e esse dado está próximo ao estudo de Barbosa (2014), o qual obteve 3,66\% de óleo essencial de alecrim utilizando o mesmo método de extração do presente estudo. Sugere-se que essa pequena 
variação nos dados esteja relacionada com as espécies em estudo e as condições de cultivo (SOUZA et al., 2014).

No tratamento a pleno sol e com sombrite a $30 \%$ de luminosidade também obtiveram 3,33\% de teor óleo essencial, ou seja, não verificou variação no rendimento de óleo essencial, como pode se observar na Figura 1.

Figura 1: Teor do óleo essencial do Rosmarinus sp. antes do tratamento e após tratamento (a pleno sol e a 30\% de luminosidade).

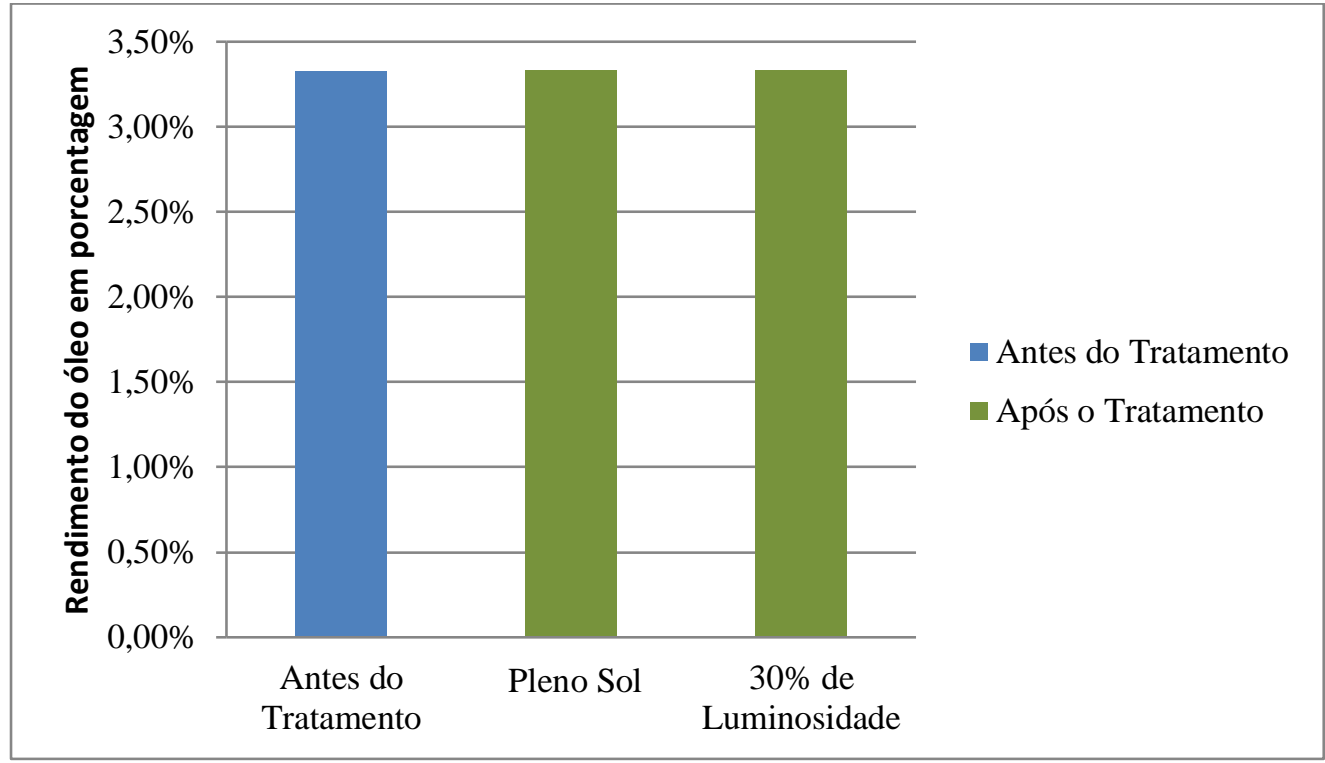

Os dados do presente estudo corroboram com o trabalho realizado por Pinto et al., (2007) com a espécie Aloysia gratissima (alfazema-do-Brasil); em seus tratamentos de sombreamento (a pleno sol, 80\% e 40\%) também não houve efeito significativo sobre o teor do óleo essencial. Também no estudo desenvolvido por Costa et al. (2010), com a espécie Ocimum selloi (manjericão) nenhum dos tratamentos de luz alteraram o rendimento do teor de óleo essencial, embora a produtividade foi maior em plantas cultivadas a pleno sol, devido a maior densidade de tricomas glandulares.

Em contrapartida, no trabalho realizado por Souza et al. (2014) o maior valor de óleo essencial de Rosmarinus officinalis L. (alecrim) foi observado nas plantas cultivadas a pleno sol do que nas plantas cultivadas sob sombreamento (50\%). Os autores justificam que esta resposta foi atribuída ao efeito da intensidade luminosa, uma vez que o alecrim é uma planta que prefere climas mais secos, ensolarados, sendo que estas condições climáticas irão determinar um melhor desenvolvimento e consequentemente uma melhor qualidade do óleo essencial. 
Portanto, esse dado difere do presente estudo. Talvez isso esteja relacionado com tempo de experimento, uma vez que o estudo de Souza et al. (2014) as mudas de Rosmarinus officinalis L. (alecrim) foram cultivas durante 4 meses.

Geralmente a produção de óleo essencial está associada com maior irradiância e taxa fotossintética superior (ARAÚJO, 2014), isso pode ser observado no estudo com Otacanthus azureus (Erva-copaíba), em que foi encontrado um maior rendimento de óleo essencial extraído das plantas cultivadas em nível de $100 \%$ de irradiância, sendo que o teor de óleo essencial da espécie em estudo teve seu valor reduzido com a diminuição do nível de luz $(50 \%$ de irradiância) (SERUDO et al., 2013). Também na espécie Lippia citriodora Lam (cidrão) a produção de óleo essencial sob os tratamentos $50 \%$ e $25 \%$ a luminosidade apresentaram menores valores que as plantas cultivadas a pleno sol (GOMES et al., 2009).

Já no trabalho de Mattana et al. (2010) com Pothomorphe umbellata (L.) Miquel quando submetidas a níveis de sombreamento (30,50 e 70\% e a pleno sol), apresentou o maior teor de óleo essencial em plantas com 30\%. Segundo os mesmos autores a 100\% de irradiação geralmente causam necrose e clorose com a fotodegradação de pigmentos, levando a uma redução da fotossíntese e um baixo rendimento de biomassa.

Fator observado no presente estudo foi o crescimento das mudas de alecrim submetidas ao tratamento com $30 \%$ de luminosidade em relação às mudas cultivadas a pleno sol. Isso corrobora com o estudo desenvolvido por Brant et al. (2009), em que espécie Melissa officinalis L. cultivada com malhas de sombreamento favoreceu o crescimento em comparação ao cultivo a pleno sol. Pacheco e Paulilo (2009) justificam que esse crescimento está relacionado com a procura do vegetal à luz solar, isso acontece quando espécies de sol são submetidas ao cultivo com sombreamento.

Segundo Meira et al. (2012) a intensidade luminosa pode influenciar no desenvolvimento vegetal, causando alterações morfofisiológicas. Martins et al., (2009) observou que nas plantas de Ocimum gratissimum (alfavaca-cravo) o cultivo a pleno sol, proporcionou maior espessura foliar e densidade de tricomas glandulares, os quais são responsáveis por produzir os óleos essenciais.

\section{CONCLUSÃO}

Não houve diferença significativa no teor de óleo essencial das plantas cultivadas a pleno sol e com as plantas cultivadas a sombreamento de $30 \%$ de luminosidade, mas devido à importância dos óleos essenciais e seus valores econômicos no Brasil é importante a 
continuidade desse estudo aumentando o tempo de tratamento, pois os óleos essenciais, em especial da espécie Rosmarinus sp. são muito utilizados na perfumaria, cosmética e na área de alimentos.

\section{REFERENCIAS BIBLIOGRÁFICAS}

AFONSO, M. S.; SANT'ANA, L. S.; MANCINI-FILHO, J. Interação entre antioxidantes naturais e espécies reativas do oxigênio nas doenças cardiovasculares: perspectivas para a contribuição do alecrim (Rosmarinus officinalis L.). Nutrire: Revista da Sociedade Brasileira de Alimentação e Nutrição, v. 35, n. 1, p. 129-148,2010.

ARAÚJO, L. L. N. Características morfofisiológicas, produção e composição de óleo essencial em folhas de tetradenia riparia (hochst) codd- lamiaceae cultivada em diferentes níveis de sombreamento. 2014. 81 f. Dissertação (Mestrando em Biodiversidade Vegetal) Instituto de Ciências Biológicas, Universidade Federal de Goiás, Goiânia.

BARBOSA, V et al. Avaliação da atividade antibacteriana do óleo essencial de Rosmarinus officinalis L. e tintura de própolis frente à bactéria causadora da acne Propionibacteriumacnes. Revista Brasileira de Plantas Medicinais, v.16, n.2, p.169-173, 2014.

BRANT, R. S et al. Crescimento, teor e composição do óleo essencial de Melissa cultivada sob malhas foto conversoras. Ciência Rural, v. 39, n. 5, p. 1401- 1407, 2009.

BRASIL. Farmacopéia Brasileira. Agência Nacional de Vigilância Sanitária. v.2, $5^{\mathrm{a}}$ ed, Brasília: Anvisa, 2010, p. 852.

CAMPOS, M. A. A; UCHIDA, T. Influência do sombreamento no crescimento de mudas de três espécies amazônicas. Pesquisa Agropecuária Brasileira, v.37, n.3, p.281-288, 2002.

CHAGAS, J. H. Crescimento de plantas, teor e composição química volátil de Mentha arvensis $\mathrm{L}$. em função da intensidade e qualidade de luz, métodos de secagem e tempos de extração.2009. 137 f. Tese (Doutorando em Fitotecnia) - Universidade Federal de Lavras, Minas Gerais.

CLEFF, M. B et al. Perfil de suscetibilidade de leveduras do gênero Cândida isoladas de animais ao óleo essencial de Rosmarinus officinalis L. Revista Brasileira Plantas Medicinais, v.14, n.1, p.43-9, 2012.

COSTA, L.C. B etal.Yield and composition of the essential oil o Ocimum selloi cultivated under colored netting. Journalof Essential Oil Research, v.22, n. 1, p.34-39, 2010.

FRESCURA, V.D et al. Compostos Fenólicos em Extratos de Rosmarinus Officinalis L. sob Cultivo Fora do Solo. Enciclopédia Biosfera, Centro Científico Conhecer, v.9, n.17, p. 755$761,2013$. 
GOMES, P. A et al. Influência do sombreamento na produção de biomassa, óleo essencial e quantidade de tricomas glandulares em cidrão (Lippia citriodora Lam) Revista Biotemas, v. 22, n. 4, p. 9-14, 2009.

MARTINS, J. R. et al. Anatomia foliar de plantas de alfavaca-cravo cultivadas sob malhas coloridas. Ciência Rural, v.39, n.1, p.82-87, 2009.

MATTANA, R.S et al. Shade level effects on yield and chemical composition of the leaf essential oil of Pothomorpheumbellata (L.) Miquel. ScientiaAgricola.v. 67, n. 4, p. 414-418, 2010.

MAY, A et al. Produção de biomassa e óleo essencial de alecrim (Rosmarinus Officinalis L.) em função da altura e intervalo entre cortes. Revista Brasileira de Plantas Medicinais, v.12, n.2, p. $195-200,2010$.

MEIRA, M.R.; MARTINS, E.R.; MANGANOTTI, S.A.Crescimento, produção de fitomassa e teor de óleo essencial de melissa (Melissa officinalis L.) sob diferentes níveis de sombreamento. Revista Brasileira de Plantas Medicinais, v.14, n.2, p.352-357, 2012.

MORAIS L. A. S. Influência dos fatores abióticos na composição química dos óleos essenciais. Horticultura Brasileira, v.27, n. 2, p. 4050- 4063,2009.

MULLER, L. Incorporação de óleo essencial de cravo em membranas de quitosana e avaliação da atividade antimicrobiana e cicatrizante. 2013, 98 f. Dissertação (Mestrando em Engenharia Química) - Universidade Federal de Santa Catarina, Florianópolis.

PACHECO, P.; PAULILO, M. T. S. Efeito da intensidade de luz no crescimento inicial de plantas de Cecropia Glazioui Snethlage (Cecropiaceae). Revista de Botânica, n. 38, p. 28-41, 2009.

PINTO, J. E. B. Pet al. Aspectos morfofisiológicos e conteúdo de óleo essencial de plantas de alfazema-do-Brasil em função de níveis de sombreamento. Horticultura Brasileira, v. 25, n. 2 p. 210-214, 2007.

PRINS, C. L et al. Efeito de fatores ambientais sobre a produção de fitomassa aérea, rendimento e composição do óleo essencial do alecrim. Centro de Ciências e Tecnologias Agropecuárias, Laboratório de Fitotecnia - Setor de Plantas Daninhas e Medicinais, 2008.

RIDEIRO, D. S et al. Avaliação do óleo essencial de alecrim (Rosmarinus officinalis L.) como modulador da resistência bacteriana. Semina: Ciências Agrárias, v. 33, n. 2, p. 687-696, 2012.

SERUDO, R. Net al. Acúmulo de matéria seca e rendimento de óleo da planta Otacanthus azureus em função da luminosidade e adubação nitrogenada. Scientia Plena, v. 9, n. 11, p 15,2013 .

SIMÕES C. M. O et al. Farmacognosia: da planta ao medicamento - 6 ed- Porto Alegre: Editora UFRGS, Florianópolis: Editorada UFSC, 2007. 
SOUZA, G. Set al. Crescimento vegetativo e produção de óleo essencial de plantas de alecrim cultivadas sob telas coloridas. Bioscience Journal, v. 30, n. 1, p. 232-239,2014.

TEIXEIRA, L. Avaliação do uso do extrato de alecrim de jardim (Rosmarinus officinalis linn) no controle do biofilme dental. 2012. 28 p. Disponível em: <http://www.odontologia.ufpr.br/bancotcc/CD_14/Lucimari\%20Teixeira.pdf.> Acesso em: 23 de fevereiro de 2015. 